\title{
THE INFLUENCE OF AN INNOVATIVE LOCOMOTOR STRATEGY ON THE PHENOTYPIC DIVERSIFICATION OF TRIGGERFISH (FAMILY: BALISTIDAE)
}

\author{
A Dornburg \\ B Sidlauskas \\ F Santini \\ L Sorenson \\ Virginia Institute of Marine Science \\ TJ Near \\ See next page for additional authors
}

Follow this and additional works at: https://scholarworks.wm.edu/vimsarticles

Part of the Aquaculture and Fisheries Commons

\section{Recommended Citation \\ Dornburg, A; Sidlauskas, B; Santini, F; Sorenson, L; Near, TJ; and Alfaro, ME, "THE INFLUENCE OF AN INNOVATIVE LOCOMOTOR STRATEGY ON THE PHENOTYPIC DIVERSIFICATION OF TRIGGERFISH (FAMILY: BALISTIDAE)" (2011). VIMS Articles. 925. \\ https://scholarworks.wm.edu/vimsarticles/925}


Authors

A Dornburg, B Sidlauskas, F Santini, L Sorenson, TJ Near, and ME Alfaro

This article is available at W\&M ScholarWorks: https://scholarworks.wm.edu/vimsarticles/925 


\title{
THE INFLUENCE OF AN INNOVATIVE LOCOMOTOR STRATEGY ON THE PHENOTYPIC DIVERSIFICATION OF TRIGGERFISH (FAMILY: BALISTIDAE)
}

\author{
Alex Dornburg, ${ }^{1,2}$ Brian Sidlauskas, ${ }^{3,4,5}$ Francesco Santini,, 6 Laurie Sorenson,, 8,9 Thomas J. Near, ${ }^{1,10,11}$ \\ and Michael E. Alfaro ${ }^{6,12}$ \\ ${ }^{1}$ Department of Ecology and Evolutionary Biology, Yale University, New Haven, Connecticut 06520 \\ ${ }^{2}$ E-mail: alex.dornburg@yale.edu \\ ${ }^{3}$ Department of Fisheries and Wildlife, Oregon State University, Corvallis, Oregon 97331 \\ ${ }^{4}$ National Evolutionary Synthesis Center, Durham, North Carolina 27705 \\ ${ }^{5}$ E-mail: brian.sidlauskas@oregonstate.edu \\ ${ }^{6}$ Department of Ecology and Evolutionary Biology, University of California, Los Angeles, California 90095 \\ ${ }^{7}$ E-mail: santini@eeb.ucla.edu \\ ${ }^{8}$ Virginia Institute of Marine Science, Gloucester Point, Virginia 23062 \\ ${ }^{9}$ E-mail: Isorenso@vims.edu \\ ${ }^{10}$ Peabody Museum of Natural History, Yale University, New Haven, Connecticut 06520 \\ ${ }^{11}$ E-mail: thomas.near@yale.edu \\ ${ }^{12}$ E-mail: michaelalfaro@ucla.edu
}

Received August 28, 2009

Accepted February 8, 2011

Innovations in locomotor morphology have been invoked as important drivers of vertebrate diversification, although the influence of novel locomotion strategies on marine fish diversification remains largely unexplored. Using triggerfish as a case study, we determine whether the evolution of the distinctive synchronization of enlarged dorsal and anal fins that triggerfish use to swim may have catalyzed the ecological diversification of the group. By adopting a comparative phylogenetic approach to quantify median fin and body shape integration and to assess the tempo of functional and morphological evolution in locomotor traits, we find that: (1) functional and morphological components of the locomotive system exhibit a strong signal of correlated evolution; (2) triggerfish partitioned locomotor morphological and functional spaces early in their history; and (3) there is no strong evidence that a pulse of lineage diversification accompanied the major episode of phenotypic diversification. Together these findings suggest that the acquisition of a distinctive mode of locomotion drove an early radiation of shape and function in triggerfish, but not an early radiation of species.

KEY WORDS: Adaptive radiation, balistiform, correlated evolution, disparity, generalized least squares, geometric morphometrics, locomotion, Tetraodontiformes. 
Functional novelty is thought to spur diversification in ecomorphological traits as a consequence of creating ecological opportunity-the chance for a lineage to exploit new niches (Schluter 2000; Gavrilets and Losos 2009; Yoder et al. 2010). However, expectations of how functional innovations influence the tempo of lineage, morphological, or functional diversification are less clear. The ecological model of adaptive radiation predicts a burst of morphological disparity and lineage diversification immediately following functional innovation as lineages fill newly available niches, then a slowing of diversification after niches fill (Schluter 2000; Rabosky and Lovette 2006a; Rabosky and Lovette 2006a,b) or a consequence of reaching functional limits or hard boundaries in morphospace (Blomberg et al. 2003; Freckleton and Harvey, 2006; Harmon et al. 2010). However, some recent studies have failed to find strong links between functional innovations and phenotypic diversification (Slater et al. 2010) or species richness (Alfaro et al. 2009a,b). Understanding why the consequences of functional innovations vary so much among case studies represents a fundamental goal in macroevolutionary biology.

Within the more than 31,000 living fish species (Eschmeyer 2010) exists a tremendous diversity in locomotor morphology and behavior including fascinating modes of swimming, gliding, walking, crawling, and underwater flight. This variation presents rich opportunities for the study of diversification following functional innovation in locomotion strategies. However, despite an explosion of interest in fish hydrodynamics enabled by increasingly accessible three-dimensional (3D) flow visualization (e.g., Mittal et al. 2006; Tytell 2006; Lauder and Madden 2006; Dabiri 2009; Lauder 2010), there have been few comparative evolutionary studies of locomotor innovation and phenotypic diversification. These studies have generally focused on the integration of locomotion with other functional systems such as feeding (Rice and Westneat 2005; Higham 2007; Collar et al. 2008) and have found functional changes in locomotion to potentially influence changes throughout the entire bauplan of a fish lineage. As locomotion is central to a fish's ecology, the question of whether evolutionary change in locomotor structure following major functional shifts follows the predictions of macroevolutionary theories such as key innovation or the ecological theory of adaptive radiation can be raised.

Triggerfish possess one of the more distinctive swimming modes within fish, using coupled oscillation or undulation of paired median fins to achieve forward thrust. This mode of locomotion, termed balistiform swimming (Sfakiotakis et al. 1999), is found in several fish groups including flatfish (Pleuronectiformes) and filefish (Monacanthidae), although the greatest diversity in fin shape is found within triggerfish. Shape diversity spans deep-bodied, large-finned species such as Melichthys niger to shallow-bodied species with high aspect ratio fins such as Canthideris maculata. Diversity in fin and body shape is thought to partially reflect divergent locomotor strategies (Lighthill and Blake 1990a; Wright 2000). Median fin oscillators rely upon strongly tapering high aspect ratio fins whereas undulators possess anteroposteriorly elongate fins of more uniform depth (Lighthill and Blake 1990a,b,c,d). However, triggerfish are not limited to this simple dichotomy of forms, and a substantial diversity of intermediate morphologies are found within triggerfish that have not been quantitatively examined in hydrodynamic studies (see Wright 2000).

Triggerfish represent a good case study from which to explore the diversification dynamics associated with functional innovation for several reasons. Theoretical models assume symmetry between dorsal and anal fins (Lighthill and Blake 1990a,b,c,d; Wright 2000; Korsmeyer et al. 2002; Loofbourrow 2009), implying that morphological evolution of the fins should be tightly correlated. Because the ratio of fin to body depth has been demonstrated to have a strong influence of the overall drag (Lighthill and Blake 1990a,d), fin and some aspects of body shape evolution should also be highly correlated. These predictions about morphological evolution in triggerfish have never been tested. Moreover, the recent reconstruction of a phylogeny and chronogram from this group (Alfaro et al. 2007; Dornburg et al. 2008) provides the framework to ask how the evolution of a major functional innovation (balistiform locomotion) influenced subsequent cladogenesis and phenotypic diversification.

Here we use a suite of phylogenetic comparative methods to ask two classes of questions about the influence of a novel form of locomotion on the evolutionary dynamics of triggerfish morphology: (1) Do triggerfish median fin and body shapes exhibit strong patterns of correlated evolution as would be expected if balistiform locomotion constrains their morphological evolution, and (2) is there evidence for a rapid or adaptive character to the diversification of triggerfish lineages, morphology, and function as would be expected if the evolution of their distinctive locomotor type catalyzed an adaptive radiation? To address those questions, we characterize fin shape, aspect ratio, and body shape in two-thirds of all extant triggerfish species and analyze patterns of morphological and functional diversification in the context of a time-calibrated molecular phylogeny.

\section{Methods}

To investigate patterns of correlated evolution between components of the triggerfish locomotor system and infer the historical processes that have helped to shape their standing biodiversity, we gathered data on morphology, function, and phylogeny. We measured morphological and functional diversity among species, constructed morphospaces to identify the most important axes of variation, and quantified the relationship between phylogeny and fin and body shape morphospace to look for evidence of lineage clustering within the phylomorphospace (Sidlauskas 2008). 


\section{QUANTIFYING PHENOTYPIC DIVERSITY OF TRIGGERFISH}

We photographed 270 adult specimens comprising 26 species of triggerfish (Appendix S1) using 8-10 megapixel digital cameras. All specimens were photographed facing left, with individual photos taken of each fish's body in addition to its dorsal and anal fins. Caudal fins could not be photographed consistently due to frequent damage and were not included in shape analysis. For each species, we aimed to digitize between 5 and 20 individuals, though for both the rare Red Sea endemic Rhinecanthus assasi and the West African Balistes punctatus only two individuals were available.

We used landmark-based geometric morphometrics (Bookstein 1991; Adams et al. 2004; Zelditch et al. 2004) to capture the shapes of the fins and body. We placed four total landmarks on the origin and insertion of the fin base and tips of the anterior and posterior rays and used sliding semi-landmarks (Bookstein 1997) to describe the curvature present along the distal margin (Fig. S1). The sliding semi-landmarks were placed by outlining each distal fin margin and resampling the curve to contain eight sliding semi-landmarks. We also placed one sliding semi-landmark along the fin base at the midpoint between the fin origin and insertion for a total of 13 fin landmarks (four fixed, nine sliding semi-landmarks). To quantify body shape, we used 27 homologous landmarks (Fig. S2) and positioned five semilandmarks by eye to better define the curves of the body at the midpoint of the following pairs of landmarks: (1) 3 and 9, (2) 10 and 12, placed along the body, (3) 14 and 15, placed along the fin-ray insertion margin, (4) 17 and 18, placed along the body, and (5) 21 and 22. All of the landmarks were placed using TpsDIG2 (Rohlf 2006).

We quantified a functional property of each fin by calculating its aspect ratio (Lighthill and Blake 1990a; Wright 2000). Each fin was outlined in TpsDIG2 (Rohlf 2006) and the area and semispan were computed (Fig. S3). Wright (2000) defined the semispan as the length from the tip of the fin to the flapping axis, drawn perpendicular to the $x$-axis (Fig. S3). Following Wright (2000), we computed fin aspect ratios for each individual as the semispan squared, divided by the total fin area. We also repeated this analysis defining aspect ratio according to Walker and Westneat (2002) as two times the length of the fin's leading edge squared, divided by the total fin area (Fig. S3). Mean aspect ratio values for each species were used in further analysis. The potential for biased error driven by allometry was assessed for both shape and mechanical data by testing for a correlation between fin or body centroid size and partial warp scores or aspect ratios using multivariate regression (Monteiro 1999) in TPSRegr (Rohlf 2003).

\section{DETERMINING THE MOST IMPORTANT AXES OF SHAPE VARIATION}

To quantify the most important axes of shape variation for each fin or body shape dataset, we used a Procrustes fit (Rohlf and Slice 1990; see also Zelditch et al. 2004) to generate a mean shape for each species and remove variation due to scaling, rotation, and translation (Zelditch et al. 2000). We then used a second Procrustes fit of the 26 consensus configurations combined with a relative warps (RWs) analysis (Rohlf 1993) in TpsRelw version 1.46 (Rohlf 2007) to generate a morphospace (a series of orthogonal eigenvectors describing the major axes of shape variation). Because we set alpha to 0, the RW analysis was mathematically equivalent to a principal components analysis of the Procrustes coordinates (Rohlf 1993). In subsequent analysis, all RW scores were multiplied by one hundred to accommodate easier interpretation (following Sidlauskas 2008).

\section{DIVERGENCE TIME ESTIMATION}

To estimate the phylogeny and timing of evolutionary divergences in triggerfish, we assembled DNA sequence data for 28 balistid species (Table S1), representing 11 of 12 genera, and comprising approximately two-thirds of described triggerfish species. Most of these data derive from Dornburg et al. (2008), although two taxa, Pseudobalistes naufragium and Sufflamen verres, were newly collected by MEA and sequenced, following the same protocols as Dornburg et al. (2008). Our study also includes three species of filefish (Monacanthidae), representing the closest sister group to the balistids (Santini and Tyler, 2003; Holcroft 2005; Alfaro et al. 2007; Dornburg et al. 2008; Yamanoue et al. 2008). We were unable to sample sufficient morphological data for adult Sufflamen lunula and Rhinecanthus verrucosus, and these two taxa were subsequently pruned from our chronogram for analyses of morphometric data.

We conducted a Bayesian relaxed-clock time calibrated phylogenetic analysis (Drummond et al. 2006) of the triggerfish using two of the same calibration age priors as Dornburg et al. (2008). The first based the divergence of the lineages giving rise to extant families Balistidae and Monacanthidae on four fossil stem balistoids dated to 35 million years (MY): Balistomorphus orbiculatus, B. ovalis, B. spinosus, and Oligobalistes robustus (Tyler and Santini 2002). We followed Alfaro et al. (2007) and assigned an upper bound of $70 \mathrm{MY}$ to this calibration that reflects the appearance of several other tetraodontiform families in addition to the first stem tetraodontiforms in the fossil record. Divergence time analyses were repeated with the recently discovered Eocene taxa Gornylistes prodigiosus used a calibration (Bannikov and Tyler 2008), although the change in the marginal posterior density of crown triggerfish ages was negligible. The second calibration age 
prior placed a normally distributed prior age constraint on the age of crown balistids based on Alfaro et al.'s (2007) analysis. These prior age calibrations reflect the fossil record of balistids as no crown balistid fossils are known to be older than the middle Miocene (Schultz 2004), whereas stem balistids date back to at least 35 MY (Tyler and Santini 2002). We omitted one calibration used in Dornburg et al. (2008). Although the fossil Balistes procapriscus has been suggested to provide a minimum age on the split between Balistes and its sister group, Pseudobalistes (Santini and Tyler 2004), analyses of molecular datasets reveal Pseudobalistes to be polyphyletic (Dornburg et al. 2008), making assignment of this calibration ambiguous.

We estimated divergence times using the concatenated data under a model of uncorrelated but log-normally distributed rates using BEAST (Drummond et al. 2006), placing a birth-death prior on rates of cladogenesis. All fossil constraints incorporated soft upper bounds to avoid artificially truncating the posterior distribution of our divergence time estimates (e.g., Yang and Rannala 2006). Analyses were run with and without data to assess the influence of the prior on the posterior distribution of age estimates (Drummond et al. 2006). We used the nucleotide data partitioning strategy identified as having the highest Bayes factor support, with support being considered as Bayes factors greater than 10 , resulting in partitioning our data by gene and codon (Kass and Raftery 1995; Brandley et al. 2005; Brown and Lemmon 2007) using the best-fit models of nucleotide substitution selected using AIC in jModelTest (Posada and Crandall 1998). We ran three independent analyses of 30 million generations and assessed convergence of the chains using Tracer 1.3 (A. Rambaut and A.J. Drummond) and AWTY (Nylander et al. 2008). The effective sample sizes (ESS) for model parameters were assessed to ensure good mixing of each chain with ESS values above 200 indicating appropriate sampling from the posterior distribution of each parameter. We further plotted the cumulative split frequency for each node between runs, assessing convergence of the chains by a stabilization of the cumulative posterior probability.

\section{LINEAGE DIVERSIFICATION}

If triggerfish experienced an ecological adaptive radiation (Schluter 2000), we predicted that species diversification rates would be highest early in the history of the clade and then slow through time as available niches filled (Schluter 2000; Rabosky et al. 2007). We initially tested this assumption using a modification of the Monte Carlo constant rates (MCCR) test (Pybus and Harvey 2000) that accounts for incomplete taxon sampling. Although the MCCR test was implemented in Dornburg et al. (2008), recent studies have found the gamma statistic of this test to be sensitive to biased, nonrandom, taxon sampling strategies, such as those employed by researchers attempting to sample all genera or functional groups (Cusimano and Renner 2010; Brock et al. 2011). As such, we implemented an extension of the MCCR test that accounts for nonrandom sampling of proportionally older splits while calculating the gamma statistic (Brock et al. 2011).

Although tests such as the MCCR test (Pybus and Harvey 2000) can detect early pulses of lineage accumulation, they cannot distinguish if this is a consequence of shifts in speciation, extinction, or alternate models such as density-dependent speciation (Rabosky and Lovette 2006a; Rabosky et al. 2007). For example, a higher than expected distribution of nodes toward the root of the tree might be the consequence of a variable rate of extinction, or could be explained by the expectations of a multirate birth-death model, and may not reflect a rapid initial radiation of species. To assess the best-fit model of lineage diversification for our data, we simultaneously compared all models using Akaike's information criterion (Akaike 1973) in LASER. To test this hypothesis, we simultaneously compared the fit of several lineage diversification models using Akaike's information criterion (Akaike 1973) in $\mathrm{R}$ using the LASER package (Rabosky 2006a,b). The pool of candidate models included the pure birth (Yule) and birth-death model of speciation originally compared by Dornburg et al. (2008) as well as seven additional models. These included two fluctuating rate models (two rate Yule, and two rate birth death), a model allowing either speciation and extinction rates to vary through time, and two nested simpler models that held either the speciation or extinction rates constant while allowing the other parameter to vary through time. Finally the candidate pool also included a lognormal and an exponential model of density-dependent lineage diversification that posit the lineage diversification rate to slow through time as the radiation progresses (Rabosky and Lovette 2006a,b). To account for the potential effects of incomplete taxon sampling on these model fitting approaches to lineage diversification, we simulated 1000 random tree topologies using APE (Paradis et al. 2004) and Geiger (Harmon et al. 2007) under a pure-birth process using the empirical speciation rate inferred under a pure-birth process. This null distribution of trees was pruned to our level of taxon sampling (28 of 42 extant species) either randomly, or using one of three strategies that preferentially pruned younger taxa that had originated during the last $50 \%, 33 \%$, or $25 \%$ of the time spanned by the phylogeny. These biased sampling strategies more accurately reflected our sampling of real triggerfish species, which was designed to sample at least one species from all major subclades and span reasonable levels of bias toward older bifurcations that may result from our empirical inclusion of all major triggerfish lineages. We assessed the fit of the lineage diversification models to this pool of pruned null trees and compared our empirical fit to the expected distribution of AIC score differences that result as a consequence of both random and nonrandom incomplete taxon sampling using the LASER package in R (Rabosky 2006b). 


\section{CORRELATED EVOLUTION BETWEEN COMPONENTS OF BALISTIFORM LOCOMOTOR MORPHOLOGY}

We tested for evidence of correlated evolution between the most important components of variation in our fin shape datasets while taking the expected covariance among traits due to phylogeny into account by using the phylogenetic generalized least squares (PGLS) method in the APE (Paradis et al. 2004) software package in R. As the model of evolution can affect this type of analysis, tests were conducted under both a Brownian (random walk) and OU (constrained) model of evolution, while comparing the AIC fit of each model (Table S2). To assess whether elements of body shape are integrated with the evolution of balistid locomotor morphology, we used a multiple regression using body shape as the dependent variable while taking the interactions of the fin shapes into account. Model fitting was conducted under both a BM and OU model of phenotypic change, with AIC values greater than four being used to select between model fits (Burnham and Anderson 2002).

We used partial least squares (PLS) (Rohlf and Corti 2000) in TPSPLS (Rohlf 2005) and MorphoJ (Klingenberg 2008) to identify pairs of axes that explain the maximum covariance between the datasets, in a manner mathematically similar to a principal components analysis emphasizing covariance as opposed to variance (Bookstein and Rohlf 2004; Zelditch et al. 2004). This method can only assess covariance between pairs of datasets so we conducted three pairs of PLS analyses for our shape data, one for each possible combination of the fin and body shape matrices. To test whether the shared covariance of the datasets was robust to the influence of phylogeny, we also extracted pairs of covarying PLS axes and tested them for significant correlated evolution using PGLS.

\section{MORPHOLOGICAL AND FUNCTIONAL DIVERSIFICATION}

Clades that have undergone an ecological adaptive radiation (Schluter 2000) are expected to partition more ecofunctional trait variation among rather than within subclades (Harmon et al. 2003). We used phylomorphospace visualization (Sidlauskas 2008) and analyses of disparity through time (Harmon et al. 2003) to investigate whether triggerfish exhibited this pattern. Phylomorphospaces combine morphometric and phylogenetic datasets to provide a visual assessment of how lineages partition available morphospace and how closely phylogenetic proximity predicts morphological similarity. We plotted the three RWs explaining the greatest percentage of total variance for each dataset (body, dorsal fin, and anal fin) in 3D morphospaces, and projected the phylogeny linking these species into the same space by reconstructing the morphological position of the internal nodes using weighted squared change parsimony. All phylomorphospaces were plotted using the Rhetenor module (Dyreson and Maddison 2003) in Mesquite (Maddison and Maddison 2008).

Assessing the relative subclade disparity among lineages has become an integral part of macroevolutionary studies focusing on how lineages occupy a morphospace (e.g., Foote 1997; Eble 2000; Valentine and Jablonski 2003; Villier and Eble 2004; Jablonski 2005). If the novelty of the triggerfish locomotive system has driven a rapid early pulse of diversification in their shape morphology, triggerfish lineages should achieve higher relative subclade disparity early in their history than would be expected under a random model of evolution. We tested this hypothesis by calculating the relative subclade disparity through time for fin aspect ratios and the most important axes of fin and body shape (Harmon et al. 2003). To assess whether triggerfish diversity patterns differed from a null model of Brownian evolution, we simulated the evolution of fin and body shape variable on the triggerfish topology using the empirical variance for each trait 1000 times and calculated the morphological disparity index (MDI) using the Geiger package (Harmon et al. 2007) in R. Negative MDI values indicate that subclades vary strongly from one another whereas positive values indicate that subclades have converged. To account for our level of incomplete taxon sampling of tipward taxa, we followed Harmon et al. (2003) and restricted our analysis of MDI values to the first $60 \%$ of the time spanned by the phylogeny.

\section{Results}

\section{DETERMINING THE MAJOR AXES OF MORPHOLOGICAL VARIATION}

Two RW axes explained approximately $76 \%$ of the total body shape change variation for triggerfish (Table 1). The first axis of body shape change described an elongation of the overall body coupled with an anteroposterior compression of the cranium (Fig. 1). The second RW axis for body shape change described a dorsoventral compression of body depth coupled with an anteroposterior elongation of the body and cranium (Fig. 1). The combined body shape data also provide evidence for substantial restructuring of the triggerfish cranium, with the dorsal slope of the cranium becoming steeper and elongating.

For the dorsal and anal fins, the first three RWs explained $90 \%$ and $93 \%$ of the overall variance for each fin shape, respectively (Table 1). For both the dorsal and anal fins, the first RW

Table 1. Percent of variance explained by the first three relative warps.

\begin{tabular}{llll}
\hline & Anal fin & Dorsal fin & Body shape \\
\hline Relative Warp 1 & 57.18 & 59.72 & 51.94 \\
Relative Warp 2 & 20.62 & 19.49 & 23.4 \\
Relative Warp 3 & 12.69 & 13.86 & 7.5 \\
\hline
\end{tabular}



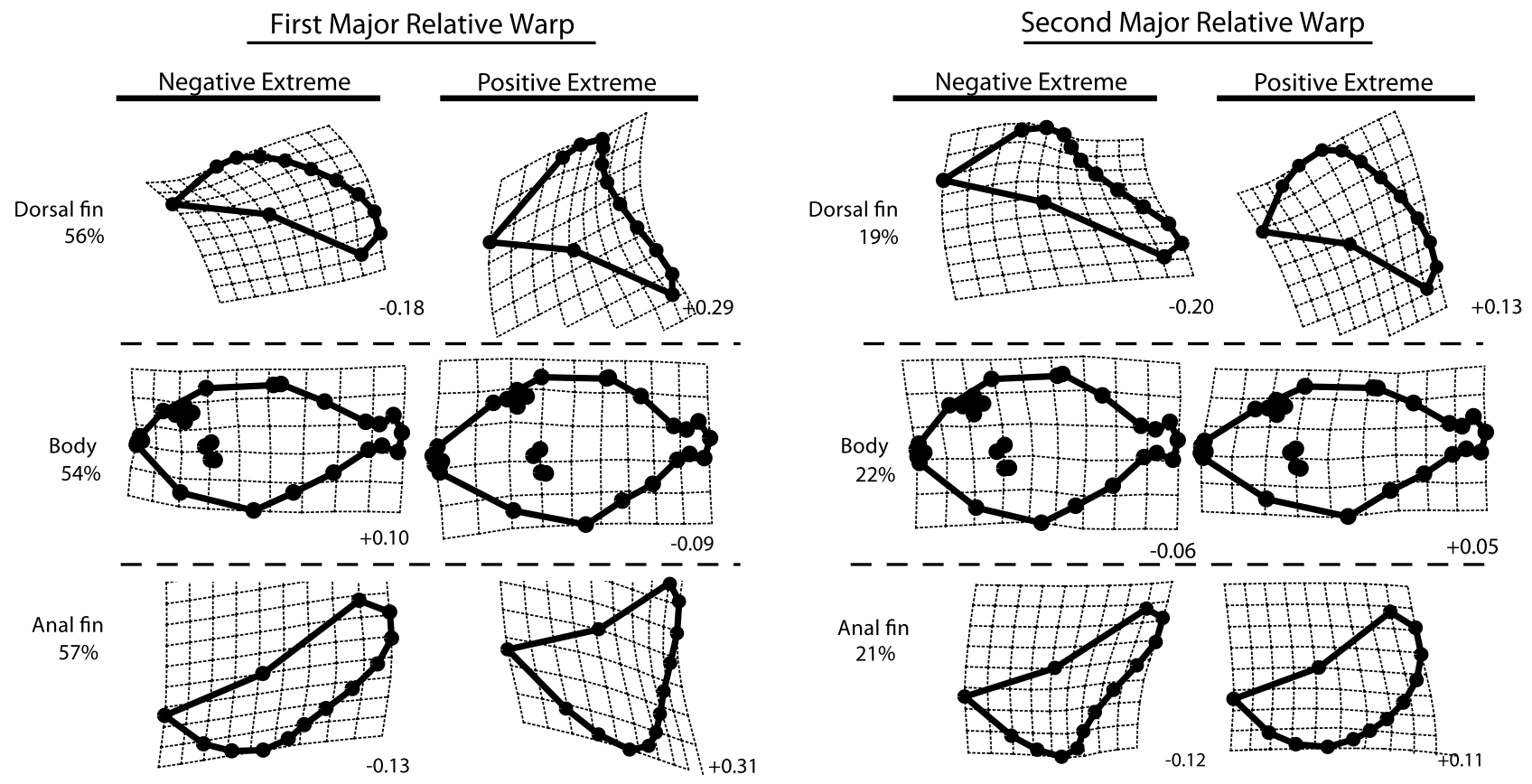

Figure 1. Dorsal fin, body shape, and anal fin shape change quantified by the first two relative warps. Positive and negative extremes represent the maximum observed phenotypic divergence on either end of the respective RW axis.

(explaining 57-60\% variance) described the change in the length of the anterior (leading edge) fin rays (Fig. 1). The second RW in both fins (explaining 19-21\% variance) described variation in the anteroposterior length of the fin coupled with a dorsal to ventral elongation of fin rays posterior to the leading edge (Fig. 1). The third RW axis (explaining 13-14\% of the variance in shape change) described a change in curvature along the distal margin of the fin (image not shown).

\section{TRIGGERFISH PHYLOGENETICS AND DIVERGENCE TIME ESTIMATION}

Our inferred phylogeny contains six major clades: (1) Canthidermis; (2) Sufflamen; (3) Rhinecanthus; (4) Abalistes; (5) Balistes; and (6) all other balistids, providing strong support for the paraphyly and polyphyly of multiple genera congruent with the findings of Dornburg et al. (2008) (Fig. 2). Our results mirror Dornburg et al. (2008), as we found Balistes to be paraphyletic, with strong support present for nested placement of Pseudobalistes fuscus and P. naufragium within this clade. There was strong support for a sister relationship between Balistoides viridescens and $P$. flavimarginatus, suggesting these genera are also not monophyletic. Our analysis revealed high support values $(\mathrm{PP}>0.95)$ for most nodes in the tree, with the exception of the three most basal divergences (Fig. 2).

We estimated a crown age of balistids of approximately 10 MY, with the $95 \%$ highest posterior density (HPD) interval spanning approximately 7-14 MY, suggesting Balistidae to be relatively young (Fig. 2). The chronogram also suggests that the stem lineages of the six major clades identified above began to diversify in the Late Miocene. Abalistes and Canthidermis appear to have split relatively rapidly from the lineage that gives rise to clades 2 and 3 ( $\sim 9 \mathrm{MY}$ ). The confidence intervals of our age estimates are all well within the bounds of the ages estimated by Dornburg et al. (2008), suggesting that the placement of the additional calibration age prior based on the fossil B. procapriscus had a minimal influence on the analysis (Table S2).

\section{PATTERNS OF TRIGGERFISH LINEAGE DIVERSIFICATION}

The rate of lineage diversification $\left(\lambda_{\mathrm{G}}\right)$ for the triggerfish was estimated at approximately 0.266 lineages per million years, based on a pure-birth (Yule) model. Similar log likelihood scores for the fit of the Yule (-4.1363) and birth-death (-4.1360) models of lineage diversification prevented us from being able to reject a pure-birth model as the underlying process for triggerfish lineage diversification. When the candidate pool of models was expanded to include seven additional models of lineage diversification, a density-dependent model of speciation was inferred to be the best fit, suggesting rates of lineage diversification to have declined over the history of triggerfish evolution. However, this pattern of declining rates of cladogenesis most likely reflects a methodological bias as our simulations designed to mimic various taxon sampling strategies demonstrated that this result is within the range of the model-fit expectations that would occur based on incomplete and nonrandom taxon sampling (Fig. 3). Similarly, accounting for nonrandom taxon 


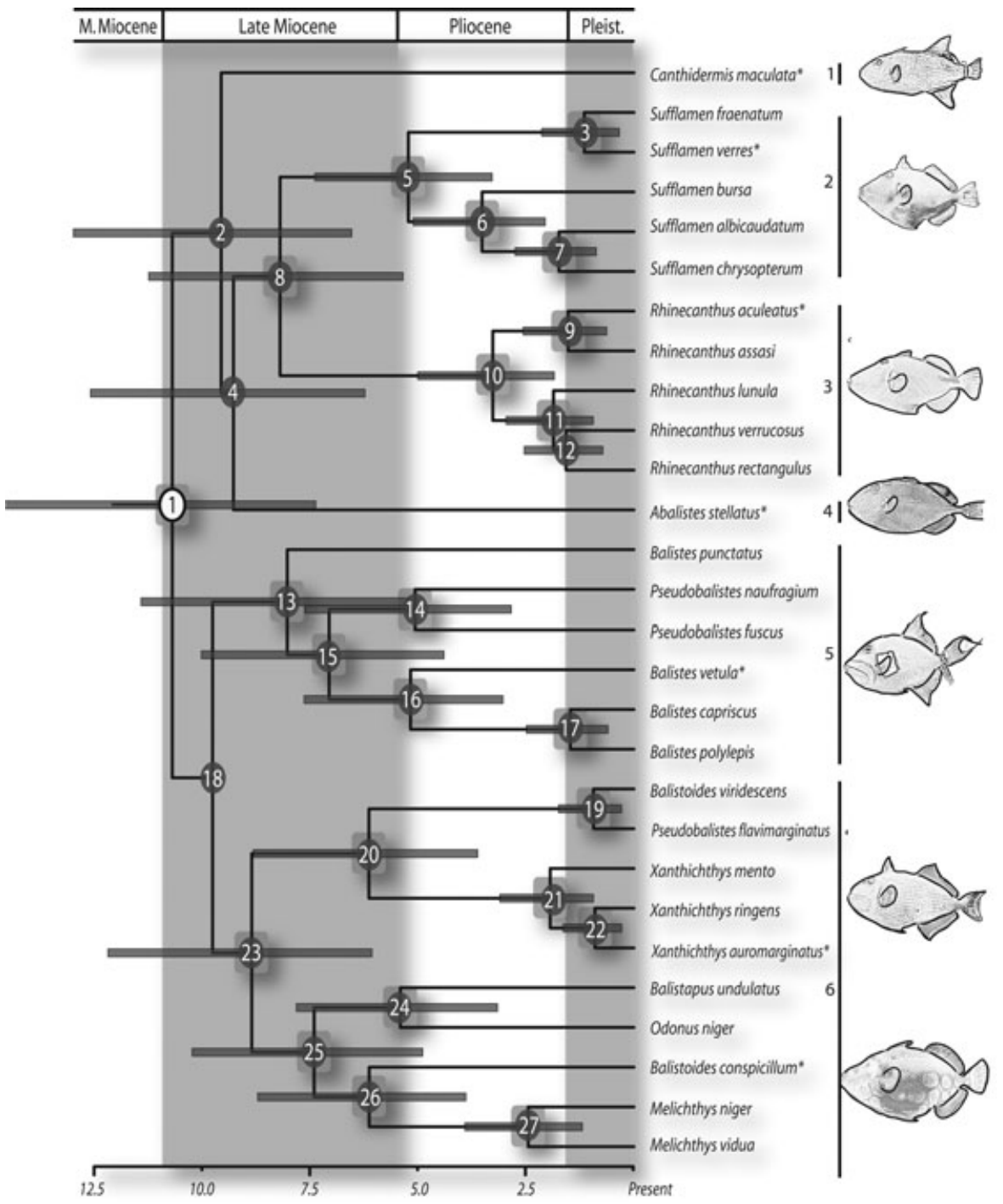

Figure 2. Consensus chronogram of triggerfish divergence times. Bars around nodes represent the $95 \%$ HPD. Shaded dark boxes at nodes indicate posterior probabilities (PP) $>0.95$, whereas numbered nodes correlate with Appendix 2 . White numbered nodes indicate the presence of prior age constraints. Nodes with PP less than 0.5 do not contain a $95 \%$ HPD on the age estimate.

sampling in the interpretation of the gamma statistic $(\gamma \sim-1.99)$ using modifications of the MCCR test (Brock et al. 2011) further demonstrated how nonrandom taxon-sampling strategies that are designed to capture older splits bias lineage diversification rate tests toward reconstructing early bursts of cladogenesis (Fig. S4).

\section{CORRELATED EVOLUTION AMONG COMPONENTS OF BALISTIFORM LOCOMOTION}

PGLS analysis provided strong evidence for correlated evolution between the two most important RWs of the dorsal fin and each of the reciprocal RWs of the anal fin (Table 2). This suggests that the heightening, elongation, or skewing of one of these fins is linked to a similar change in the other $(0.00001<P<0.05)$. These results were identical under both a Brownian and constrained
(OU) model of phenotypic evolution. We observed congruent patterns when analyzing pairs of PLS axes while accounting for the expected covariance due to shared phylogenetic history using PGLS (Table 3). Further, plotting dorsal fin shape RW1 against anal fin shape RW1 reveals a striking pattern of tight correlation between the two RWs, confirming that the two traits to have undergone correlated evolution (Fig. S5).

Multiple regression analysis provided strong evidence for a pattern of integrative evolution between the interacting fin shapes and the first major body shape warp under both a BM and OU model $\left(r^{2} \sim 0.71\right)$. Conversely, we obtained no significant correlation between the second RW of body shape and the interactions of the anal and dorsal fin shapes $\left(r^{2} \sim 0.3\right)$, supporting a decoupling of the evolution of dorsalventral compression of the body from the elongation and lengthening of fin shapes. 

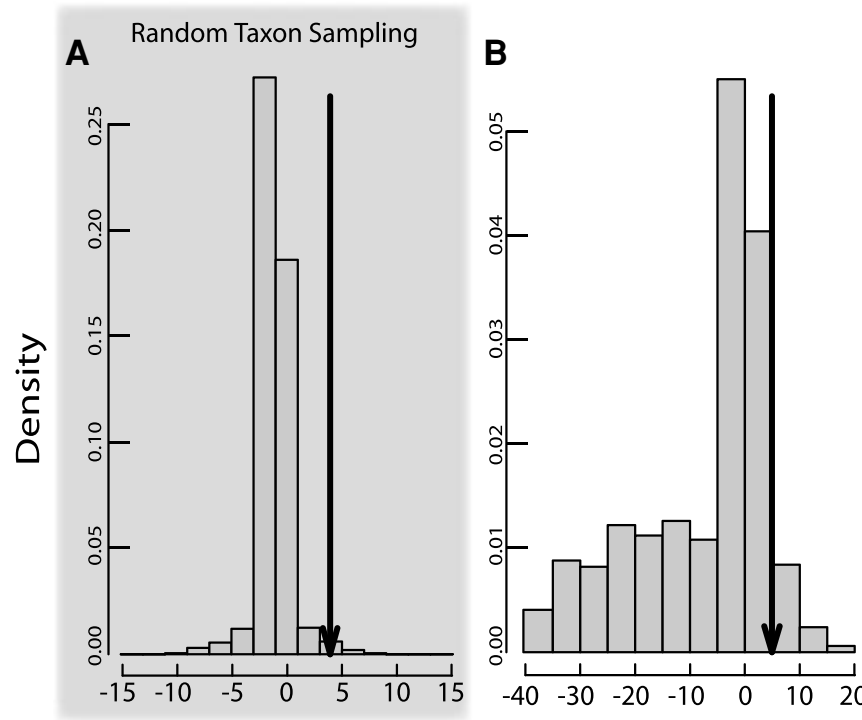

C Non-Random Taxon Sampling

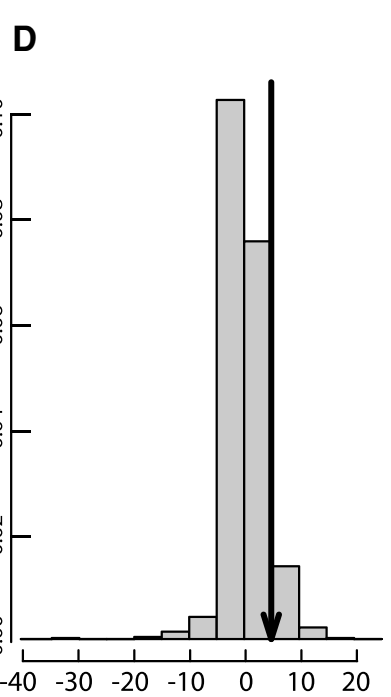

\section{Null Distribution of $\triangle \mathrm{AIC}$}

Figure 3. Distribution of AIC differences $(\triangle A I C)$ generated by fitting a Yule and exponential density-dependent models of speciation (DDX) to pruned trees simulated under a pure birth process. Positive $\triangle A I C$ indicate preferred fits for the DDX model. Dark arrows represent empirical fit of triggerfish data. (A) Expected $\triangle$ AIC distribution generated by randomly sampling Yule trees $(P \sim 0.01)$; (B) Expected $\Delta$ AIC distribution generated by preferentially pruning lineages from the more recent $50 \%$ of each tree's length $(P<0.07)$; (C) Expected $\Delta$ AIC distribution generated by preferentially pruning lineages from the more recent $33 \%$ of each tree's length (P 0.09); and (D) Expected $\triangle$ AIC distribution generated by preferentially pruning from the more recent $25 \%$ of each tree's length $(P \sim 0.09)$.

\section{MORPHOLOGICAL AND FUNCTIONAL DIVERSIFICATION}

We find evidence of phylogenetic clustering for both the fin and body-shape data, with subclades occupying distinct regions of morphospace (Fig. 4A-C). Additionally, plotting the bodyshape RW1 against either the dorsal or anal fin RW1 (Fig. 4D and E) reveals that nearly every major clade occupies a position in morphospace that is divergent from that of its sister clade. The only major qualitative difference between these fig-

Table 2. Testing for correlated evolution between fin shapes.

\begin{tabular}{cll}
\hline & $\begin{array}{l}\text { First major warp } \\
\text { dorsal fin } \\
\text { R-squared/ } \\
P \text {-value }\end{array}$ & $\begin{array}{l}\text { Second major warp } \\
\text { dorsal fin } \\
\text { R-squared/ } \\
P \text {-value }\end{array}$ \\
\hline $\begin{array}{c}\text { First major warp } \\
\text { anal fin }\end{array}$ & $\mathbf{0 . 5 7 / < \mathbf { 0 . 0 0 1 }}$ & $0.08 />0.10$ \\
$\begin{array}{c}\text { Second major warp } \\
\text { anal fin }\end{array}$ & $\mathbf{0 . 1 5} /<\mathbf{0 . 0 5}$ & $\mathbf{0 . 1 6 / < 0 . 0 5}$ \\
\hline
\end{tabular}

Results of phylogenetic generalized least squares test for correlation between fin shapes. Results in bold indicate variables explaining significant variation in the dependent variable. Presented are results based on a null expectation of BM with the dorsal fin as the dependent variable, results based on an OU process or model of OLS, or with the anal fin as the dependent variable were nearly identical. ures is the wide separation of members of lineage 5, identified as "Balistes" sensu Dornburg et al. (2008) and the genus Canthidermis in the anal-fin shape/body shape phylomorphospace (Fig. 4E).

Plots of the fin aspect ratio phylomorphospace (Fig. 4F) indicate that major functional groups of triggerfish evolved early in the group's history, supporting the hypothesis of early functional diversification. When combined with Figure 2 and the plots of dorsal fin morphospace (Fig. 4A), the fin aspect ratio visualizations reveal that triggerfish partition the mechanospace between five major functional groups. The majority of triggerfish taxa occupy

Table 3. Testing for correlated evolution among principal PLS axes.

\begin{tabular}{llr}
\hline Dataset & Axes & $P$-value/slope \\
\hline Dorsal fin vs. body shape & first & $\mathbf{0 . 0 0 0 9 / 0 . 2 2 3}$ \\
Dorsal fin vs. body shape & second & $\mathbf{0 . 0 0 8 8 / 0 . 1 4 4}$ \\
Anal fin vs. body shape & first & $\mathbf{0 . 0 0 0 2 / 0 . 3 5 3}$ \\
Anal fin vs. body shape & second & $\mathbf{0 . 0 0 1 6 / 0 . 2 4 4}$ \\
Dorsal fin vs. anal fin & first & $\mathbf{< 0 . 0 0 0 1 / 1 . 1 4}$ \\
Dorsal fin vs. anal fin & second & $\mathbf{< 0 . 0 0 0 1 / 1 . 0 1}$ \\
\hline
\end{tabular}

Results from the phylogenetic generalized least squares test for correlated evolution between major axes of variation from the partial least squares analysis. Bold $\boldsymbol{P}$-values indicate significant results. 
A

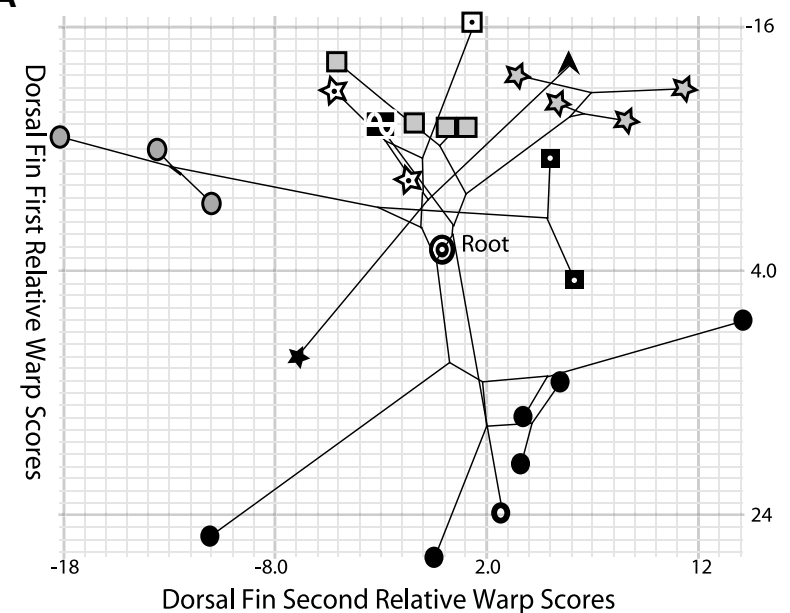

C

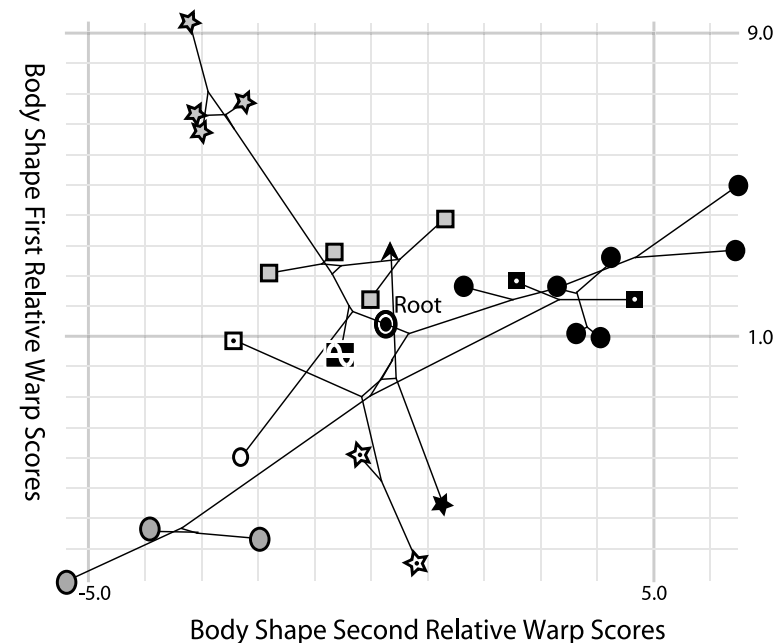

E

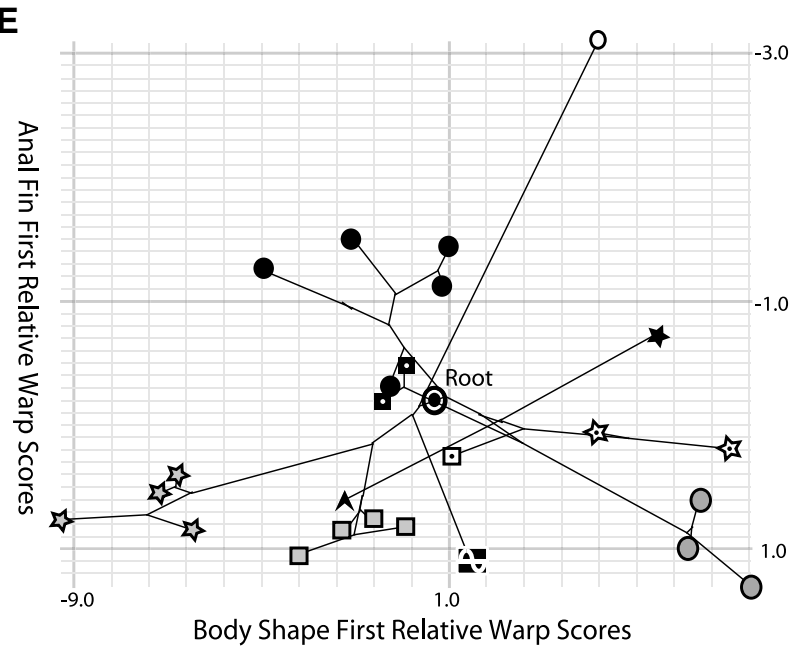

B

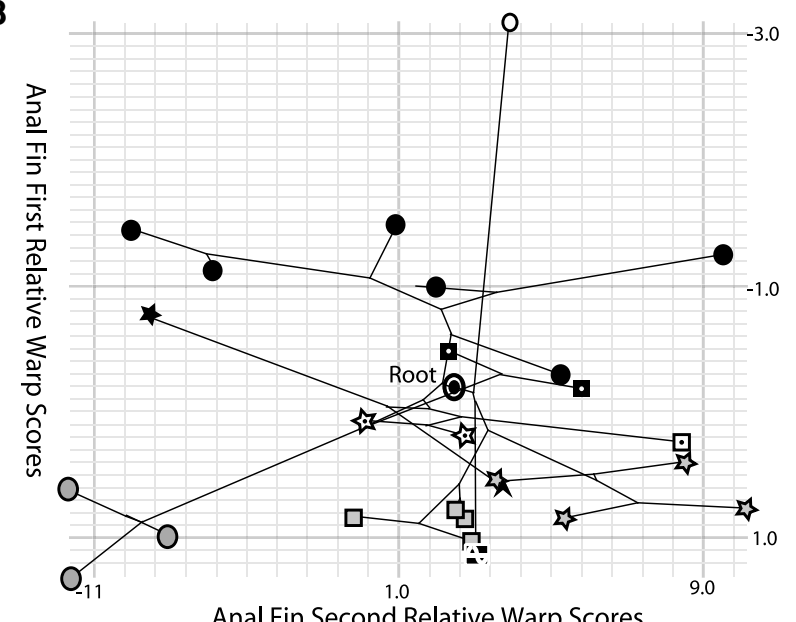

D

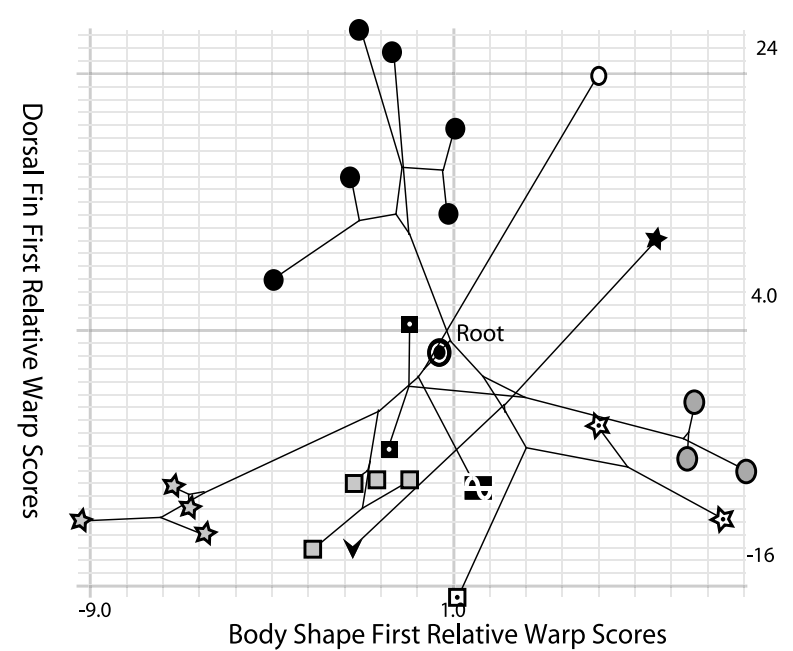

$F$

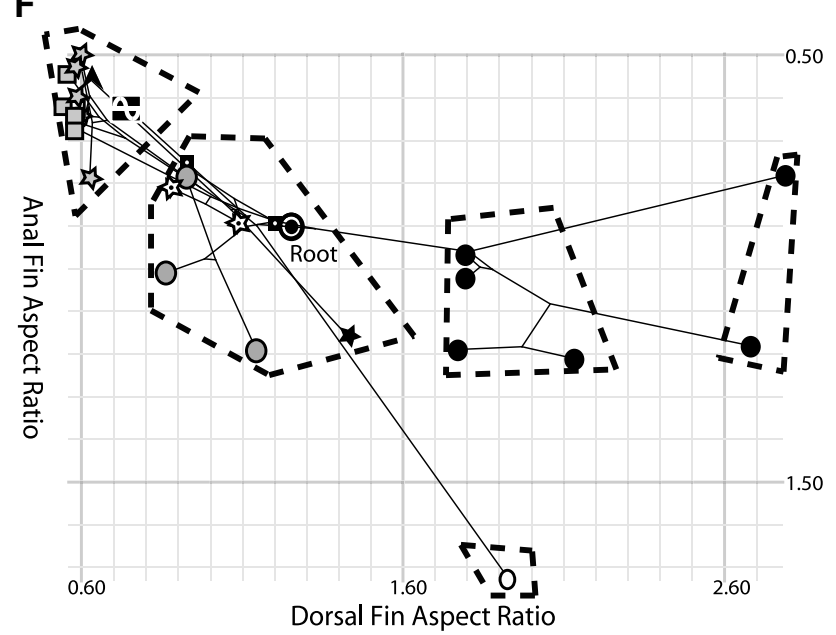

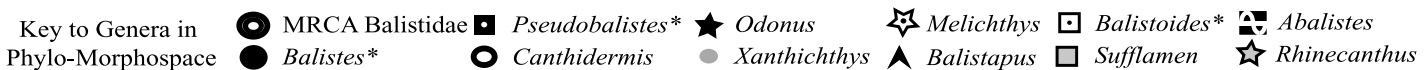

Figure 4. Phylomorphospace visualizations for the major relative warps. Comparisons of how lineages occupy different morphospaces: (A) The first and second dorsal fin RWs; (B) the first and second anal fin RWs; (C) the first and second body shape RWs; (D) comparison between the first dorsal fin RW and the first body shape RW; (E) the first anal fin and the first body shape RW; (F) the aspect ratio of the dorsal fin and the aspect ratio of the anal fin. *Genus names follow the classification proposed in Dornburg et al. (2008). 
regions of functional space characterized by low aspect ratios, a state that evolved early in their history. Transitions between high and low aspect ratios within groups are rare, with the exception of members of Balistes sensu Dornburg et al. (2008) (lineage 5, Fig. 2). This lineage occupies the largest region of mechanospace, and comprises nearly $50 \%$ of the observed functional diversity of these fish (Fig. 4F). This partitioning of the morphospace through time is reflected in the disparity through time plots of fin shape, body shape, and aspect ratio evolution (Fig. 5). In each case, we reveal a departure from a null Brownian simulation, with negative MDI statistics indicating that early crown triggerfish partitioned the morphospace following the evolution of balistiform locomotion.

\section{Discussion}

Three lines of evidence suggest that the functional demands of balistiform locomotion have influenced triggerfish shape evolution. First, the evolution of the most important axes of anal and dorsal fin shapes is highly integrated. Second, key aspects of median fin and body shape have coevolved in triggerfish. Third, triggerfish appear to have explored the limits of their modern phenotypic and functional diversity early in their history. These results support the idea that the majority of shape diversity observed in modern triggerfish reflects an underlying early diversification of function and are consistent with the expectation that evolutionary novelty spurs functional diversification. However our analysis of lineage diversification fails to find a corresponding early pulse of speciation, suggesting morphological and lineage diversification to have been decoupled during triggerfish evolution. Thus, the history of triggerfish diversification does not conform to the expectations of the classical adaptive radiation model.

\section{PATTERNS OF CORRELATED SHAPE EVOLUTION}

The results of every test for correlated evolution that we performed (Tables 2 and 3) as well as the observed linear relationship between the RWs of the median fins in phylomorphospace (Fig. S7) strongly suggest that the two major locomotor structures of triggerfish, the dorsal and anal fins, evolved in lockstep. Further, the phylomorphospace visualizations of aspect ratio (Fig. 4F) show that mechanical evolution in triggerfish median fins closely reflects patterns of morphological evolution (Fig. 4A and B). The visualizations also imply ancestral crown triggerfish to have had a medium aspect ratio (Fig. 4F), with a subsequent history of most lineages converging on low and medium aspect ratios (Upper left corner, Fig. 4F). Low aspect ratio fins are correlated with increased maneuverability and are associated with fish that remain in close proximity to reefs, whereas higher aspect ratios are associated with increased water column usage and also shallower habitats with more wave energy (Fulton et al. 2005). These expec- tations suggest two functional hypotheses to explain the tightly correlated evolution of dorsal and anal fin shape and function: (1) balistiform swimmers may be functionally constrained to achieve propulsive forces using coupled symmetrical fins, or (2) there may be a developmental constraint in which the dorsal and anal fin belong to the same module. These hypotheses are not mutually exclusive. The potential lift generated by the dorsal and anal fins may constrain these fins to evolve in tandem for efficient maneuverability in the water column, whereas developmental modules could also shape the development of the fish's underlying musculature and skeletal elements (e.g., Mabee et al. 2002). Sorenson (2007) recently found a startling degree of symmetry between the underlying dorsal and anal fin structural elements in Rhinecanthus rectangulus, supporting the idea of a developmental module underlying the primary locomotor components of triggerfish.

Our analyses also suggest that major features of body shape evolve in tandem with fin shape. For example, we find that elongate bodies are coupled with rounder, less-sigmoidal fins (Table 3, Fig. S6 left panel). Fish with this body plan tend to be more reef associated (Lieske and Myers 2001; Bean et al. 2002) suggesting this to be an efficient body plan for maneuvering complex 3D environments. Triggerfish lineages associated with pelagic and open environments are also characterized by high aspect ratio fins and bulbous reduced crania (Fig. S6). This suggests modifications of the cranial morphology to be a potentially important and understudied aspect of the hydrodynamics of balistiform locomotion.

\section{WHAT DROVE FUNCTIONAL AND MORPHOLOGICAL DIVERSIFICATION IN TRIGGERFISH?}

The morphological disparity indices and the phylomorphospace visualizations show that triggerfish colonized nearly all of their presently occupied morphospace early in their radiation. All axes of shape and functional change are partitioned among rather than within lineages early in the history of the group (Fig. 4), and the accumulation of disparity among lineages appears to occur rapidly, mostly during the first 1-2 MY of the group's history. This pattern is consistent with recent adaptive radiation models in which early lineages invade disparate regions of morphospace whereas later lineages subpartition initially colonized regions (Schluter 2000; Harmon et al. 2003). However, triggerfish depart from these models in one important sense: they do not show the expected pattern of initially rapid lineage diversification.

Although we recover a pattern of elevated early cladogenesis while assuming random taxon sampling, the results of simulations that account for nonrandom taxon sampling clearly demonstrate the sensitivity of these test statistics to violations of this assumption (e.g., Cusimano and Renner 2010; Brock et al. 2011). Two scenarios might explain the apparent patterns of uncoupled 

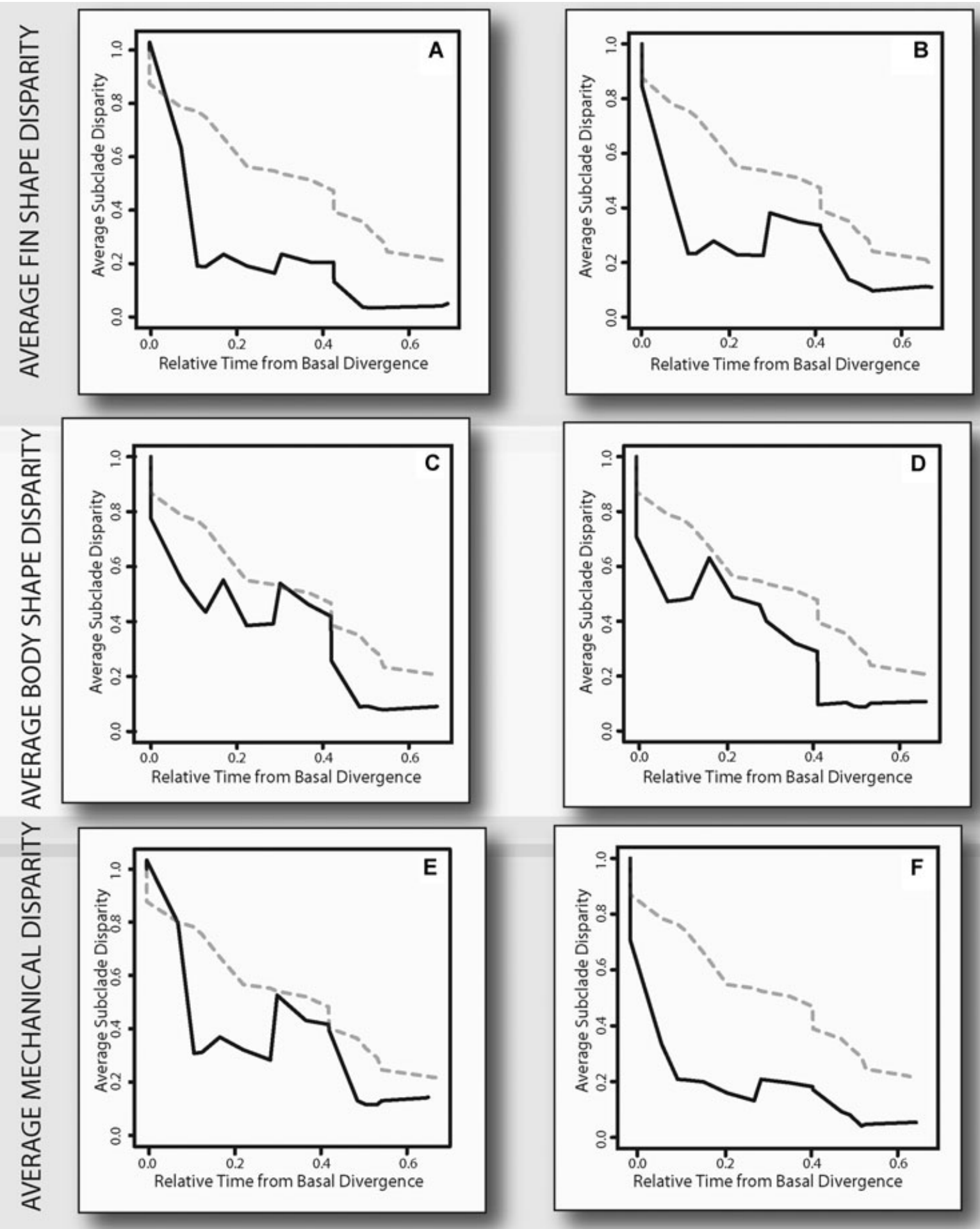

Figure 5. Disparity through time plots for anal fin shape RW1 (A), Dorsal fin shape RW1 (B), body shape RW1 (C), body shape RW2 (D), anal fin aspect ratio $(E)$, dorsal fin aspect ratio $(E)$. The solid curve represents the empirically inferred pattern subclade disparity through time. The dashed curve represents the median of the Brownian simulations. All plots were generated using the Geiger (Harmon et al. 2007) package in $\mathbf{R}$. 
lineage diversification and morphological evolution in triggerfish. It is possible that these processes were in fact coupled, but the elevated rates of extinction eroded the signature of early rapid lineage diversification (Quental and Marshall 2009; Rabosky 2009). We regard this scenario as unlikely for two reasons. First it requires morphologically disparate subclades to resist total elimination even while extinction acts within them to reduce richness. This is not a frequent outcome of standard models of high lineage turnover, which tend to eliminate early branching lineages entirely and generate very young crown clades (Raup 1985; Sidlauskas 2007). Second, some simple violations of the assumption of random extinction, such as the heritability of extinction rates, are expected to bias methods for testing diversification rates toward inferring an early pulse of diversification even when the true diversification rate remains constant (Rabosky 2009). In spite of that potential bias, we fail to recover evidence of initially rapid diversification. Thus, although it cannot be ruled out completely, we believe it unlikely that triggerfish experienced an invisible burst of speciation shortly after evolving balistiform locomotion.

A second possibility is that triggerfish adaptive diversification reflects a process where phenotypic diversification is decoupled from cladogenesis. Although the most prevalent model of ecological adaptive radiation (e.g., Schluter 2000) predicts that these processes will be linked, the most cited examples of this phenomenon are clades with restricted geographic distributions such as islands or lakes (Day and Wilkinson 2006; Seehausen 2006, Losos 2009; Johnson et al. 2009), quite unlike the broad species ranges that characterize most triggerfish. In widespread marine species, long-distance larval dispersal (e.g., Palumbi 1994, Bellwood et al. 2006) might strongly alter the expected dynamics of linked species and phenotypic diversification following evolutionary innovation by decreasing the probability of localized ecological speciation (e.g., Rocha and Bowen 2008; Budd and Pandolfi 2010). Instead, a constant rate of allopatric or peripatric speciation that is governed by physical processes and therefore unaffected by functional innovation would provide the dominant processes generating new lineages (see also, Budd and Pandolfi 2010). After the rise of each new lineage, secondary contact and character displacement (rather than ecological speciation) could then act as the primary pump driving phenotypic diversification into newly available niches (e.g., Price 2010). This model would yield an initial steady increase in disparity at a rate governed by the background rate of speciation. As the accumulating lineages filled niches over time, the rate of character displacement following instances of secondary sympatry in new lineages would slow, until all niches opened by the original innovation were filled. Thus, the eventual braking of the morphological diversification rate remains the same as in the classic adaptive radiation model.

We suggest that triggerfish may have evolved under this alternative model of diversification, with balistiform locomotion serving as the functional innovation catalyzing subsequent morphological diversification. As in the classic model of adaptive radiation, the origin of balistiform locomotion likely presented the potential for ancestral triggerfish to evolve novel fin and body shape combinations, partly as a consequence of the locomotor innovation providing access to novel ecological opportunities. However, as described above, this innovation in and of itself would not alter the background rate of species formation, as any potential for sympatric ecological diversification would be impeded by the presence of long-distance dispersal. Species formation would have instead been dominated by allopatry/peripatry and the primary control on morphological diversification would have been the background rate of isolation and secondary contact between lineages. Once the novel niches were filled, new species would still arise at the unchanged background rate of allopatric or parapatric speciation, but the rate of morphological diversification would decline and daughter species, having no open paths across the adaptive landscape, would tend to resemble their ancestors.

Although speculative, this model would explain the apparent constant rate of species diversification throughout the history of triggerfish and the inferred burst of morphological diversification at the base of their phylogeny. Such a model might also explain why many freshwater fish species radiations appear to be consistent with the traditional model of ecological radiation (e.g., Barbour 1973; Witte 1984; Hunt et al. 1997; Alesandrini and Bernardi 1999; Seehausen 2002; Day and Wilkinson 2006; Seehausen 2006), although species rich tropical marine fish families often are not (Santini et al. 2009; Alfaro et al. 2009a). Continued investigations of widespread tropical marine radiations will help reveal whether the decoupling of cladogenesis and morphological change observed in the triggerfish reflects the typical condition in coral reef fish groups. Filefish (Monacanthidae) that are balistiform swimmers with long-range dispersal that also share a close affinity with triggerfish, offer one excellent opportunity to test that hypothesis, but a full exploration should target groups with varied styles of locomotion and dispersal. Such future studies will aid in the conceptual development of adaptive radiation theory and determine if the lack of tropical coral reef fish species flocks reflects a lack of looking for them or fundamental differences in the evolutionary phenomena that shape biodiversity in tropical marine versus freshwater habitats.

\section{ACKNOWLEDGMENTS}

Thanks to K. P. Maslenikov at the Burke Museum, A. Suzumoto at the Bishop Museum, J. G. Lundberg, M. Sabaj-Perez, and K. R. Luckenbill at the Philadelphia Academy of Natural Sciences, R. F. Feeney and J. A. Seigel at the LA County Museum, G. Watkins-Colwell at the Yale Peabody Museum, M. A. Rogers and K. Swagel at the Field Museum, and K. E. Hartel at the Museum of Comparative Zoology for providing access to collection specimens. Thanks to J. A. Moore and G. Watkins-Colwell 
for help photographing specimens. Thanks to J. Beaulieu, J. Oliver, M. C. Brandley, G. Thomas, G. Marroig, and an anonymous reviewer for their comments and reviews on earlier versions of this manuscript. Fieldwork was conducted with the help of J. A. Hernandez Ugalde, SINAC, and CONAGEBIO. We would also like to thank the Donoghue and Near lab groups at Yale University for helping with various aspects of this project. $\mathrm{AD}$ received support for portions of this project from a Project Deepfin student exchange grant and an NSF undergraduate research in Biology and Mathematics fellowship (UBM 0531870). MEA was funded by NSF DEB 0918748 and IOS 0819009. BLS was supported on a postdoctoral fellowship from the National Evolutionary Synthesis Center (NSF EF0423641) and by the Oregon State University Research Office and College of Agricultural Sciences.

\section{LITERATURE CITED}

Adams, D., F. L. Rohlf, and D. Slice. 2004. Geometric morphometrics: ten years of progress following the 'revolution'. Ital. J. Zool. 71:5-16.

Akaike, H. 1973. Information theory as an extension of the maximum likelihood principle. Pp. 267-281 in B. N. Petrov and F. Csaki, eds. Second annual symposium on information theory. Akademi Kiado, Budapest.

Alesandrini, S., and G. Bernardi. 1999. Ancient species flocks and recent speciation events: what can rockfish teach us about cichlids (and vice versa)? J. Mol. Evol. 49:814-818.

Alfaro, M. E., C. D. Brock, and F. Santini. 2007. Do reefs drive diversification in marine fish? Examples from the pufferfishes and their allies. Evolution 61:2104-2126.

Alfaro, M. E., C. D. Brock, B. Banbury, and P. C. Wainwright. 2009a. Does evolutionary innovation in pharyngeal jaws lead to rapid lineage diversification in labrid fish? BMC Evol. Biol. 9:255.

Alfaro, M. E., F. Santini, C. D. Brock, H. Alamillo, A. Dornburg, D. L. Rabosky, G. Carnevale, and L. J. Harmon. 2009b. Nine exceptional radiations plus high turnover explain species diversity in jawed vertebrates. Proc. Natl. Acad. Sci. USA 32:13410-13414.

Bannikov, A. F., and J. C. Tyler. 2008. A new genus and species of triggerfish from the middle Eocene of the northern Caucasus, the earliest member of the balistidae (Tetraodontiformes). Paleontol. J. 42:615-620.

Barbour, C. D. 1973. A biogeographical history of Chirostoma (Pisces: Atherinidae): a species flock from the Mexican Plateau. Copeia 1973:533-556.

Bean, K., G. P. Jones, and M. J. Caley. 2002. Relationships among distribution, abundance, and microhabitat specialization in a guild of coral reef triggerfish (family Balistidae). Mar. Ecol-Prog. Ser. 233:263-272.

Bellwood, D. R., P. C. Wainwright, C. J. Fulton, and A. S. Hoey. 2006. Functional versatility supports coral reef biodiversity. Proc. R. Soc. Lond. B 273:101-107.

Blomberg, S., T. Garland, and A. Ives. 2003. Testing for phylogenetic signal in comparative data: behavioral traits are more labile. Evolution 57:717745.

Bookstein, F. L. 1991. Morphometric tools for landmark data. Cambridge Univ. Press, Cambridge, UK.

. 1997. Morphometric tools for landmark data: geometry and biology. Cambridge Univ. Press, Cambridge, UK.

Bookstein, F. L., and F. J. Rohlf. 2004. From "mathematical dissection of anatomies" to morphometrics: a twenty-first-century appreciation of Charles Oxnard. Pp. 378-390 in F. Anapol, R. Z. German, and N. G. Jablonski, eds. Shaping primate evolution: form, function, and behavior. Cambridge Univ. Press, Cambridge.

Brandley, M., A. Schmitz, and T. W. Reeder. 2005. Partitioned Bayesian analyses, partition choice, and phylogenetic relationships of scincid lizards. Syst. Biol. 54:373-390.
Brock, C. D., L. J. Harmon, M. E. Alfaro. 2011. Testing for temporal variation in diversification rates when sampling is incomplete and nonrandom. Syst. Biol. In press.

Brown, J., and A. Lemmon. 2007. The importance of data partitioning and the utility of Bayes factors in Bayesian phylogenetics. Syst. Biol. 56:643655.

Budd, A. F., and J. M. Pandolfi. 2010. Evolutionary novelty is concentrated at the edge of coral species distributions. Science 328:1558-1561.

Burnham, K. P., and D. R. Anderson. 2002. Model selection and multimodel inference, a practical information-theoretic approach. Springer, New York.

Collar, D. C., P. C. Wainwright, and M. E. Alfaro. 2008. Integrated diversification of locomotion and feeding in labrid fishes. Biol. Lett. 4:84-86.

Cusimano, N., and S. S. Renner. 2010. Slowdowns in diversification rates from real phylogenies may not be real. Syst. Biol. 59:458-464.

Dabiri, J. O. 2009. Optimal vortex formation as a unifying principle in biological propulsion. Ann. Rev. Fluid Mech. 41:17-33.

Day, J. J., and M. Wilkinson. 2006. On the origin of the Synodontis catfish species flock from Lake Tanganyika. Biol. Letts. 2:548-552.

Dornburg, A., F. Santini, and M. Alfaro. 2008. The influence of model averaging on clade posteriors: an example using the triggerfishes (family balistidae). Syst. Biol. 57:905-919.

Drummond, A. J., S. Y. W. Ho, M. J. Phillips, and A. Rambaut. 2006. Relaxed phylogenetics and dating with confidence. PLoS Biol. 4:e88.

Dyreson, E., and D. R. Maddison. 2003. Rhetenor package for morphometrics for the Mesquite System. version 1.0. Available at http://mesquiteproject.org (accessed August 24, 2008).

Eble, G. 2000. Contrasting evolutionary flexibility in sister groups: disparity and diversity in Mesozoic atelostomate echnoids. Paleobiology 26:5679.

Eschmeyer, W. N. ed. 2010. Catalog of Fishes electronic version. 2010 Available at http://research.calacademy.org/ichthyology/ catalog/fishcatmain.asp (accessed October 25, 2010).

Foote, M. 1997. The evolution of morphological diversity. Annu. Rev. Ecol. Syst. 28:129-152.

Freckleton, R., and P. Harvey. 2006. Detecting non-Brownian trait evolution in adaptive radiations. PLoS Biol. 4:e373.

Fulton, C. J., D. R. Bellwood, and P. C. Wainwright. 2005. Wave energy and swimming performance shape coral reef fish assemblages. Proc. R. Soc. Lond. B 272:827-832.

Gavrilets, S., and J. B. Losos. 2009. Adaptive radiation: contrasting theory with data. Science 323:732-737.

Harmon, L. J., J. A. Schulte, J. B. Losos, and A. Larson. 2003. Tempo and mode of evolutionary radiation in iguanian lizards. Science 301:961964.

Harmon, L., J. Weir, C. D. Brock, and W. Challenger. 2007. GEIGER: investigating evolutionary radiations. Bioinformatics 24:129-131.

Harmon, L. J., J. B. Losos, J. Davies, R. G. Gillespie, J. L. Gittleman, W. B. Jennings, K. Kozak, M. A. McPeek, F. Moreno-Roark, and T. J. Near. 2010. Early bursts of body size and shape evolution are rare in comparative data. Evolution 64:2385-2396.

Higham, T. E. 2007. The integration of locomotion and prey capture in vertebrates: morphology, behavior, and performance. Integr. Comp. Biol. 47:82-91.

Holcroft, N. I. 2005. A molecular analysis of the interrelationships of tetraodontiform fishes (Acanthomorpha: Tetraodontiformes). Mol. Phylogenet. Evol. 34:525-544.

Hunt, D. M., J. Fitzgibbon, S. J. Slobodyanyuk, J. K. Bowmaker, and K. S. Dulai. 1997. Molecular evolution of the cottoid fish endemic to Lake Baikal deduced from nuclear DNA evidence. Mol. Phylogenet. Evol. $8: 415-422$. 
Jablonski, D. 2005. Evolutionary innovations in the fossil record: the intersection of ecology, development and macroevolution. J. Exp. Zool. B 304B:504-519.

Johnson, M. A., L. J. Revell, and J. B. Losos. 2009. Behavioral covergence and adaptive radiation: effects of habitat use on territorial behavior in Anolis lizards. Evolution 64:1151-1159.

Kass, R. E., and A. E. Raftery. 1995. Bayes factors. J. Am. Stat. Assoc. 90:773-795.

Klingenberg, C. P. 2008. MorphoJ. Faculty of Life Sciences, University of Manchester, UK. Available at http://www.flywings.org.uk/ MorphoJ_page.htm (accessed August 24, 2008).

Korsmeyer, K. E., J. F. Steffensen, and J. Herksin. 2002. Energetics of median and paired fin swimming, body, and caudal fin swimming, and gait transition in parrotfish (Scarus schlegeli) and triggerfish (Rhinecanthus aculeatus). J. Exp. Biol. 205:1253-1263.

Lauder, G. V., and P. G. A. Madden. 2006. Learning from fish: kinematics and experimental hydrodynamics for roboticists. Int. J. Automat. Comput. 4:325-335.

Lauder, G. V. 2010. Swimming hydrodynamics: ten questions and the technical approaches needed to solve them. Anim. Locom. 1:3-15.

Lieske, E., and R. Myers. 2001. Coral reef fishes. Princeton Univ. Press, Princeton, NJ.

Lighthill, J., and R. Blake. 1990a. Biofluiddynamics of balistiform and gymnotiform locomotion. Part 1. Biological background, and analysis by elongated-body theory. J. Fluid Mech. 212:183-207.

- 1990b. Biofluiddynamics of balistiform and gymnotiform locomotion. Part 2. The pressure distribution arising in two-dimensional irrotational flow from a general symmetrical motion of a flexible flat plate normal to itself. J. Fluid Mech. 213:1-10.

. 1990c. Biofluiddynamics of balistiform and gymnotiform locomotion. Part 3. Momentum enhancement in the presence of a body of elliptic cross-section. J. Fluid Mech 213:11-20.

. 1990d. Biofluiddynamics of balistiform and gymnotiform locomotion. Part 4. Short-wavelength limitation on momentum enhancement. J. Fluid Mech. 213:21-28.

Losos, J. B. 2009. Lizards in an evolutionary tree: ecology and adaptive radiation of anoles. Univ. of California Press, Berkeley, CA.

Loofbourrow, H. 2009. Hydrodynamics of balistiform swimming in the Picasso triggerfish, Rhinecanthus aculeatus. Masters thesis. Univ. of British Columbia, Vancouver.

Mabee, P. M., P. L. Crotwell, N. C. Bird, and A. C. Burke. 2002. Evolution of median fin modules in the axial skeleton of fishes. J. Exp. Zool. 294:77-90.

Maddison, W., and D. Maddison. 2008. Mesquite: a modular system for evolutionary analysis. Version 2.5.

Mittal, R., H. Dong, M. Bozkurttas, G. V. Lauder, and P. G. A. Madden. 2006. Locomotion with flexible propulsors II: computational modeling and analysis of pectoral fin swimming in sunfish. Bioinsp. Biomimet. 1:S35-S41.

Monteiro, L. 1999. Multivariate regression models and geometric morphometrics: the search for causal factors in the analysis of shape. Syst Biol. 48:192-199.

Nylander, J. A. A., J. C. Wilgenbusch, D. L. Warren, and D. L. Swofford. 2008. AWTY (are we there yet?): a system for graphical exploration of MCMC convergence in Bayesian phylogenetics. Bioinformatics 24:581583.

Palumbi, S. R. 1994. Genetic Divergence, Reproductive Isolation, and Marine Speciation. Annu. Rev. Ecol. Syst. 25:547-572.

Paradis, E., J. Claude, and K. Strimmer. 2004. APE: analyses of phylogenetics and evolution in $\mathrm{R}$ language. Bioinformatics 20:289290.
Posada, D., and K. A. Crandall. 1998. MODELTEST: testing the model of DNA substitution. Bioinformatics (Oxford) 14:817-8.

Price, T. D. 2010. The roles of time and ecology in the continental radiation of the Old World leaf warblers (Phylloscopus and Seicercus). Phil. Trans. R. Soc. B 365:1749-1762.

Pybus, O. G., and P. H. Harvey. 2000. Testing macro-evolutionary models using incomplete molecular phylogenies. Philos. Trans. R. Soc. Lond. B 267:2267-2272.

Quental, T. B., and C. R. Marshall. 2009. Extinction during evolutionary radiations: reconciling the fossil record with molecular phylogenies. Evolution 63:3158-3167.

Rabosky, D. L. 2006a. Likelihood methods for detecting temporal shifts in diversification rates. Evolution 60:1152-1164.

- 2006b. LASER: a maximum likelihood toolkit for detecting temporal shifts in diversification rates from molecular phylogenies. Evol. Bioinform. Online 2:257-260.

Rabosky, D., and I. Lovette. 2006a. Density-dependent diversification in North American wood warblers. Proc. R. Soc. Lond. B. 275:2363-2371.

Rabosky, D., S. Donnellan, A. Talaba, and I. Lovette. 2007. Exceptional among-lineage variation in diversification rates during the radiation of Australia's largest vertebrate clade. Proc. R. Soc. Lond. B 274:29152923.

- 2006b. Explosive evolutionary radiations: decreasing speciation or increasing extinction through time? Evolution 62:1866-1875.

Rabosky, D. L. 2009. Heritability of extinction rates links diversification patterns in molecular phylogenies and the fossil record. Syst. Biol. 58:629640 .

Raup, D. M. 1985. Mathematical models of cladogenesis. Paleobiology 11:4252.

Rice, A. R., and M. W. Westneat. 2005. Coordination of feeding, locomotor and visual systems in parrotfishes (Teleostei: Labridae). J. Exp. Biol. 208, 3503-3518.

Rocha, L. A., and B. W. Bowen. 2008. Speciation in coral reef fishes. J. Fish Biol. 72:1101-1121.

Rohlf, F., and D. Slice. 1990. Extensions of the procrustes method for the optimal superimposition of landmarks. Syst. Zool 39:40-59.

Rohlf, F. J. 1993. "Relative Warp Analysis and an Example of its Application to Mosquito Wings." Pp. 132-159 in L. F. Marcus, E. Bello, and A. GarciaValdecasas, eds. Contributions to morphometrics. Museo Nacional de Ciencias Naturales, Madrid.

. 2003. Bias and error in estimates of mean shape in morphometrics. J. Hum. Evol. 44:665-683.

2005. Geometric morphometrics simplified. Review of "Geometric Morphometrics for Biologists: a primer”. Trends Ecol. Evol. 20:1314.

2006. TPS series. Department of Ecology and Evolution, State University of New York, Stony Brook, NY. Available at http://life.bio.sunysb.edu/morph/.

- 2007. TPS software series. Department of Ecology and Evolution, State University of New York, Stony Brook.

Rohlf, F. J., and M. Corti. 2000. The use of two-block partial least-squares to study covariation in shape. Syst. Biol. 49:740-753.

Santini, F., and J. C. Tyler. 2003. A phylogeny of the families of fossil and extant tetraodontiform fishes (Acanthomorpha, Tetraodontiformes), Upper Cretaceous to recent. Zool. J. Linn. Soc. 139:565-617.

. 2004. The importance of even highly incomplete fossil taxa in reconstructing the phylogenetic relationships of the Tetraodontiformes (Acanthomorpha: Pisces). Integr. Comp. Biol. 44:349-357.

Santini F., L. J. Harmon, G. Carnevale, and M. E. Alfaro. 2009. Did genome duplication drive the origin of teleosts? A comparative study of diversification in ray-finned fishes. BMC Evol. Biol. 9:194. 
Schluter, D. 2000. The ecology of adaptive radiation. Oxford Univ. Press, Oxford.

Schultz, O. 2004. A triggerfish (Osteichthyes: Balistidae: Balistes) from the Badenian (Middle Miocene) of the VIenna and the Styrian Basin (Central Paratethys). Annalen des Naturhistorischen Museums in Wien 106 A:345-369.

Seehausen, O. 2002. Patterns in fish radiation are compatible with Pleistocene desiccation of Lake Victoria and 14600 year history for its cichlid species flock. Proc. R. Soc. Lond. B 269:491-497.

- 2006. African cichlid fish: a model system in adaptive radiation research. Proc. R. Soc. Lond. B 273:1987-1998.

Sfakiotakis, M., D. Lane, and J. Davies. 1999. Review of Fish Swimming modes for aquatic locomotion. IEEE J. Oceanic Eng. 24:237252.

Sidlauskas, B. 2007. Testing for unequal rates of morphological diversification in the absence of a detailed phylogeny: a case study from characiform fishes. Evolution 61:299-316.

- 2008. Continuous and arrested morphological diversification in sister clades of characiform fish: a phylomorphospace approach. Evolution 62:3135-3156.

Slater, G. J., S. A. Price, F. Santini, and M. E. Alfaro. 2010. Diversity versus disparity and the radiation of modern cetaceans. Proc. R. Soc. Lond. B 277:3097.

Sorenson, L. 2007. Examining morphological characteristics of triggerfish: a three-dimensional reconstruction of skeletal and muscular features of Rhinecanthus rectangulus. J. Young Investigators 16:3.

Tyler, J. C., and F. Santini. 2002. Review and reconstruction of the tetraodontiform fishes from the Eocene of Monte Bolca, Italy, with comments on related Tertiary taxa. Studi e Rieche sui Giacimenti Terziari di Bolca, Museo Civico di Storia Naturale di Verona 9:47-119.

Tytell, E. D. 2006. Median fin function in bluegill sunfish, Lepomis macrochirus: streamwise vortex structure during steady swimming. J. Exp. Biol. 209:1516-1534.

Valentine, J. W., and D. Jablonski. 2003. Morphological and developmental macroevolution: a paleontological perspective. Int. J. Dev. Biol. 47:517522.

Villier, L., and G. Eble. 2004. Assessing the robustness of disparity estimates: the impact of morphometric scheme, temporal scale, and taxonomic level in spatangoid echinoids. Paleobiology 30:652-665.

Walker, J. A., and M. W. Westneat. 2002. Performance limits of labriform propulsion and correlates with fin shape and motion. J. Exp. Biol. 205:177-187.

Witte, F. 1984. Ecological differentiation in Lake Victoria haplochromines: comparison of cichlid species flocks in African lakes. Pp. 155-167 in A. A. Echelle and I. Kornfield, eds. Evolution of fish species flocks. Univ. of Maine at Orono Press, Orono.

Wright, B. 2000. Form and function in aquatic flapping propulsion: morphology, kinematics, hydrodynamics, and performance of the triggerfishes (Tetraodontiformes: balistidae). Ph.D. thesis. University of Chicago, Chicago.

Yamanoue, Y., M. Miya, K. Matsuura, H. Sakai, M. Katoh, and M. Nishida. 2008. Unique patterns of pelvic fin evolution: a case study of balistoid fishes (Pisces: Tetraodontiforms) based on whole mitochondrial genome sequences. Mol. Phylogenet. Evol. 50:179-189.

Yang, Z., and B. Rannala. 2006. Bayesian estimation of species divergence times under a molecular clock using multiple fossil calibrations with soft bounds. Mol. Biol. Evol. 23:212-226.

Yoder, J. B., S. DesRoches, J. M. Eastman, L. Gentry, W. K. W. Godsoe, T. Hagey, D. Jochimsen, B. P. Oswald, J. Robertson, B. A. J. Sarver, et al. 2010. Ecological opportunity and the origin of adaptive radiations. J. Evol. Biol. 23:1581-1596.

Zelditch, M., H. Sheets, and W. Fink. 2000. Spatiotemporal reorganization of growth rates in the evolution of ontogeny. Evolution 54:1363-1371.

Zelditch, M., D. Swiderski, D. Sheets, and W. Fink. 2004. Geometric morphometrics for biologists: a primer. Elsevier Acad. Press, San Diego, CA.

Associate Editor: G. Marroig

\section{Supporting Information}

The following supporting information is available for this article:

Figure S1. Landmarks and sliding semi-landmarks used for fin shape analysis.

Figure S2. Landmarks and sliding semi-landmarks used for body shape analysis.

Figure S3. Calculation of aspect ratio (Taken from Wright 2000).

Figure S4. Calculation of the null gamma statistic based on Pybus and Harvey's (2000) Monte Carlo constant rates test.

Figure S5. First and second partial least squares axes summarizing major axes of covariance between anal and dorsal fin shapes.

Figure S6. First and second partial least squares axes summarizing major axes of covariance between dorsal fin and body shapes.

Figure S7. Comparison between the first dorsal fin and the first anal fin RWs.

Table S1. Sequences and Genbank numbers used in this study.

Table S2. AIC scores for phenotypic models of evolution in PGLS.

Table S3. Distribution of node ages.

Table S4. AICc scores for models of morphological evolution.

Appendix S1. Location of specimens examined for this study.

Supporting Information may be found in the online version of this article.

Please note: Wiley-Blackwell is not responsible for the content or functionality of any supporting information supplied by the authors. Any queries (other than missing material) should be directed to the corresponding author for the article. 